\title{
Testing patients during seizures: a European consensus procedure developed by a joint taskforce of the ILAE - Commission on European Affairs and the European Epilepsy Monitoring Unit Association
}

Sándor Beniczky ${ }^{1}$, Miri Neufeld ${ }^{2}$, Beate Diehl ${ }^{3}$, Judith Dobesberger ${ }^{4}$, Eugen Trinka ${ }^{4}$, Ruta Mameniskiene ${ }^{5}$, Sylvain Rheims $^{6}$, Antonio Gil-Nagel ${ }^{7}$, Dana Craiu ${ }^{8}$, Ronit Pressler ${ }^{9}$, David Krysl $^{10}$, Angelina Lebedinsky ${ }^{2}$, Laura Tassi $^{11}$, Guido Rubboli ${ }^{12}$, Philippe Ryvlin ${ }^{13}$

1. Department of Clinical Neurophysiology, Danish Epilepsy Center, Dianalund, and Aarhus University, Aarhus, Denmark

2. The EEG and Epilepsy Unit, Department of Neurology, Tel Aviv Medical Center, Israel

3. National Hospital for Neurology and Neurosurgery, Departments of Clinical Neurophysiology and Clinical and Experimental Epilepsy, University College London, Institute of Neurology, Queen Square, London, UK

4. Department of Neurology, Christian-Doppler-Klinik, Paracelsus Medical University, and Centre for Cognitive Neuroscience, Salzburg, Austria.

5. Clinic of Neurology and neurosurgery, Faculty of medicine, Vilnius University; Dept. of neurology, Vilnius University Hospital Santariškių klinikos, Vilnius, Lithuania

6. Department of Functional Neurology and Epileptology, Hospices Civils de Lyon and Lyon 1 University, Lyon, France 
7. Department of Neurology, Epilepsy Program, Hospital Ruber Internacional, Madrid. Spain

8. "Carol Davila” University of Medicine, Department of Clinical Neurosciences (No.6), Pediatric Neurology Clinic, Alexandru Obregia Hospital, Bucharest, Romania

9. Department of Clinical Neurophysiology, Great Ormond Street Hospital, London, UK; Clinical Neuroscience, UCL Institute of Child Health, London, UK

10. Department of Clinical Neurophysiology, Department of Neurology, Sahlgrenska University Hospital; Institute of Neuroscience and Physiology at Sahlgrenska Academy, University of Gothenburg, Gothenburg, Sweden.

11. "Claudio Munari” Epilepsy Surgery Centre, Niguarda Hospital, Milano, Italy

12. Danish Epilepsy Center and University of Copenhagen, Denmark

13. Department of Clinical Neurosciences, CHUV, Lausanne, Switzerland, and European Epilepsy Monitoring Association, Lyon, France

Running title: Ictal testing battery

Key Words: Epilepsy Monitoring Unit, feasibility, postictal, seizures, semiology, testing 


\section{SUMMARY}

There is currently no international consensus procedure for performing comprehensive peri-ictal testing of patients in the epilepsy monitoring units (EMUs). Our primary goal was to develop a standardized procedure for managing and testing patients during and after seizures in EMUs. The secondary goal was to assess whether it could be implemented in clinical practice (feasibility). A taskforce was appointed by the ILAE - Commission on European Affairs and the European Epilepsy Monitoring Unit Association, to develop a standardized ictal testing battery (ITB) based on expert opinion and experience with various local testing protocols. ITB contains a comprehensive set of 10 items that evidence the clinically relevant semiological features, and it is adaptive to the dynamics of the individual seizures. The feasibility of the ITB was prospectively evaluated on 250 seizures from 152 consecutive patients in 10 centers. ITB was successfully implemented in clinical practice in all 10 participating centers and was considered feasible in $93 \%$ of the tested seizures. ITB was not feasible for testing seizures of very short duration. 


\section{INTRODUCTION}

Long-term video-EEG monitoring is a useful diagnostic tool that provides detailed electro-clinical information to characterize seizures for accurate diagnosis and classification of patients with epilepsy ${ }^{1-4}$. Video recordings, synchronized with EEG (scalp or invasive), contain clinically relevant semiological features that occur during seizures and in the postictal period ${ }^{5}$. Many of these features can only be captured when the EMUstaff is able to test patients for various cognitive, behavioral, sensory and motor functions ${ }^{5}$. Although a recent European survey on current practices in EMUs showed that $91 \%$ of EMUs perform ictal or postictal testing, there was no standardized procedure, and many EMUs lack local guidelines for testing patients ${ }^{6}$.

In order to increase the clinical quality of data recorded in the EMUs and to facilitate multi-center studies, the International League Against Epilepsy - Commission on European Affairs (ILAE-CEA) and the European Epilepsy Monitoring Unit Association (EEMA) appointed a taskforce to develop a standardized procedure for managing and testing patients in EMU.

We present the standardized ictal testing battery (ITB) developed by the European taskforce, and evaluation of its feasibility, during implementation in clinical practice, in 10 participating centers.

\section{METHODS}

Development of the consensus procedure for managing and testing patients during and after seizures in EMU

We searched peer-reviewed papers indexed in PubMed using various combinations of the following keywords: (behavioral OR behavioural), (testing OR test OR interview OR assessing), (seizure OR ictal OR postictal), (battery OR protocol). We only found two published testing batteries for assessing patients in the EMU. The aim of both batteries was to assess impaired consciousness related to epileptic seizures. The "ictal 
bedside neuropsychological test procedure", developed by epileptologists in Germany (Bonn and Bielefeld) assessed orientation, speech and memory, but it was not standardized throughout the study period ${ }^{7}$. The Responsiveness in Epilepsy Scale (RES) was developed and then modified by epileptologists at Yale University School of Medicine; a score was calculated for the degree of impairment of consciousness ${ }^{8-9}$.

The European taskforce developed a consensus procedure, based on expert opinion and on the experience of the taskforce members with local testing batteries that had been used in the participating centers. Although these local testing batteries showed considerable similarities, there were differences in the order of presentation (timing) of the test items, and most centers had some "specific" additional items. Through an iterative process, the consensus test battery was refined until all members of the taskforce accepted the final version. We aimed at finding an appropriate balance between comprehensiveness and feasibility. The test covers many features and adapts to the dynamic of epileptic seizures according to their duration, clinical characteristics and time of occurrence, in order to optimize the clinical information inferred from the testing.

Besides ictal testing, at times difficult to perform especially in brief seizures, the battery includes specific items for postictal testing, as well as interictal testing to determine the patient's baseline. When necessary, items were modified for pediatric patients. Since the staff testing patients in the EMUs is also responsible for optimal documentation using video recordings and for patients' safety, we also included reminders about these aspects.

In addition to the detailed description of the testing battery, the taskforce designed a one-page summary with keywords, to serve as a reminder for the EMU staff.

Implementation in clinical practice 
The consensus testing-battery was translated from English into Danish, French, German, Hebrew, Lithuanian, Romanian, Spanish and Swedish. The short summary was laminated and placed in the patientrooms, along with boxes containing the test-items or laminated sheets with photos of the test items. In the participating EMUs, the staff was trained to perform the consensus testing procedure. The training included explanation of the meaning / aim of the battery items, and role-playing where some participants simulated seizures, while others tested them.

Trained epileptologists with experience in evaluating video-EEG recordings assessed the feasibility of the testing battery, while reviewing the video-EEG monitoring. Whenever the testing procedure could be successfully carried out during the ictal or postictal phase, the battery was considered feasible.

The feasibility of the testing battery was evaluated on 250 seizures, from 152 consecutive patients (76 female), in 10 centers. The study was approved by the ethics committee, and patients and caregivers were informed about the procedure prior to monitoring. Patients who gave their informed consent and had seizures in the EMU during the census period were included. Exclusion criteria were: seizures that remained unnoticed during the monitoring; testing the patients using a battery different from the consensus procedure.

Seizure duration was determined from the earliest ictal EEG or clinical sign (whichever was first) until the last clinical or EEG evidence for seizure activity (whichever was last). Seizures were classified according to the ILAE criteria $^{10}$.

\section{RESULTS}

Consensus procedure for managing and testing patients during seizures in EMU

The taskforce developed the following procedure: 
A box with test items (toothbrush, pen, key, comb, photos of objects) and a laminated copy of the testing protocol to be placed in the patients' rooms, in advance. For younger children: teddy, toy car, photos of animals like cat, dog, cow, horse (supporting information 1).

Before or at admission to the EMU, the patients are informed about the testing procedure. The patients (or parents / caregivers) are instructed to push the "patient event" button when they feel that a seizure is starting.

\section{During seizure}

Before the ictal testing, at seizure-start:

Make sure the camera is focused on the patient, and avoid standing in front of the camera.

At the bedside: attend to any immediate safety concerns first! Ensure there is adequate light for the camera in the room. Pull the covers off the patient to optimize video recordings, respecting the patients' decency.

Observe the patient whether he/she is pale or has facial flushing, whether he/she sweats or has piloerection, sialorrhea, minimal jerks or eye deviation. Say loudly (into the microphone) if you notice any of these changes, which are discrete and usually cannot be observed on the video. If these phenomena come later during the seizure, say loudly into the camera when you notice them.

Testing the responsiveness:

Greet (verbally) the patient and say the patient's first name to draw the patient's attention. If the patient does not react, touch (or pinch slightly) the arm. If the patient is able to answer, ask him/her to describe the subjective manifestation, if any. 
Testing speech comprehension and ictal palsy:

Say: "Lift arms!" Give only verbal command without visual clues (without showing). If the patient does not follow commands, demonstrate the movements and give visual clues for the patients suggesting that he/she should repeat this. If the patient does not follow, try to shake hands with the patients; for children: ask "give me five" or wave.

Verbal function:

Give two unrelated test words: "Please repeat and remember the following words" (e.g. "horse, table" or "dog, red" or "bed, toy"). Ask the patient to repeat the words.

If the patient is not reacting at all, return to testing the responsiveness.

Questions about orientation:

What is your name? Where are you? What date is it today/ what day of the week / time of day? For children: Where is your mum/ dad? Where is your toy?

Verbal memory:

Does the patient remember the two test words and/or the commands given?

Testing for anomia and visual memory:

Show the test items from the box, and ask the patient to name them. If the patient can't say the names of the test items, please ask the patient to show, what they are used for. When testing children, ask what noise 
/ sound they make. If the patient can't say anything at all, ask the patient to stick out his / her tongue; if not following the verbal command, show the patient what he/she has to do. Ask the patient to remember one test item.

Other cognitive functions:

Ask the patient to count. If over 6 years: ask the patient to read and write.

Ask the patient whether he / she remembers the test object. If the patient can't say it, present three items (including the one shown earlier) and ask the patient which one was shown before.

Touch the patient for testing the muscular tonus especially when surface EMG channels are not part of the recording.

For sensory or visual auras, perform double-stimulus testing (supporting information 2).

If the seizure is still going on, repeat the testing.

General remarks: if the patient does not follow an instruction, just continue with the next step. Repeat testing later on, if the seizure still is going on.

\section{Postictal period}

Does the patient remember the course of the seizure, and/or that he/she have had a seizure?

Can the patient recall the test-words and the test-object? If the patient can't say the name of the test object, present 3 items (including the one shown earlier) and ask the patient which one was shown before. 
Testing for speech comprehension and Todd's palsy: lift arms; lift legs. If the patient is not following verbal commands, show the movements. If not following commends even after visual clues, elevate both arms; test also the muscular tonus.

Testing for anomia and for orientation (the same procedures as during seizure testing).

Ask the patient to describe the seizure. Ask whether the patient experienced any subjective symptoms (aura). For visual aura: ask the patient to draw the visual aura. Ask the patient or caregivers to confirm this is a habitual seizure.

Optional item: after generalized tonic-clonic seizures: test for Babinski (plantar) reflex. If it is pathological on one side, note on which side. If it is pathological on both sides, check it every minute and note on which side it persists for a longer time.

Continue testing until the patient returns to normal.

\section{Interictal testing}

For comparison, perform testing during an interictal period, using the same items as for testing during seizures, for patients with abnormal baseline cognitive function. If the patient has had a seizure prior to the interictal testing, one has to wait at least one hour after offset of the postictal period.

Figure 1 shows the one-page summary, with keywords, that serves as a reminder for the personnel in the EMU.

\section{$\underline{\text { Implementation in clinical practice }}$}

The testing battery was successfully implemented in all the participating centers. 
Patients were aged 1 - 87 years (mean 31.2, median 30 years), and included 31 children under 10 years (12.4\%). Twenty-one patients had mental retardation (8.4\%). Each patient had $1-6$ seizures in the EMU (mean: 1.6 seizure / patient). Duration of the recorded seizures was between $0.1-6486 \mathrm{~s}$ (mean= $179 \mathrm{~s}$; median=77 s). Testing was started $1-649 \mathrm{~s}$ after the seizure start (mean=30.4 s; median= $16 \mathrm{~s}$ ).

Most of the seizures (226) were focal. Thirty-five of them evolved to bilateral tonic-clonic seizures. Seizure onset was localized in the temporal lobe (91), frontal lobe (59), parietal lobe (18), occipital lobe (6) and insula (1). It was not possible to localize seizure onset in 51 seizures. Ten patients had primary generalized seizures: absence (2), primary generalized tonic-clonic seizure (5) and myoclonic jerks (3). In one case it was not possible to classify whether the seizure was focal or generalized. Thirteen seizures were psychogenic nonepileptic seizures.

Fifteen of the 250 seizures were excluded: in seven cases the seizures were not noticed and patients were not tested (neither in the ictal nor in the postictal period); in eight cases the testing was not performed according to the consensus procedure, but according to the local battery.

Of the remaining 235 seizures, the testing battery was not considered feasible in 17 (7\%). All these seizures had very short duration (myoclonus, short absence seizures and short focal seizures) accounting for the failure to perform testing. Overall, the battery was considered feasible in 218 out of the 235 seizures (i.e. $93 \%$ of the included seizures).

The duration of the interictal testing, including all items, ranged between 2 and 9 minutes, depending mainly on the age and cognitive status of the patients, but also on the skills of the examiner. 


\section{DISCUSSION}

The European taskforce developed a consensus procedure for managing and testing patients during and after seizures in EMU. The battery aims at demonstrating the semiological features that can occur during the ictal and postictal period ${ }^{5}$. It also includes interictal testing to establish the baseline level for each patient. In addition, there are items reminding the personnel about patient-safety, optimizing video-EEG recordings, observing and reporting discrete changes that might not be evident on video-recordings (like pallor, flushing, sweating, piloerection, sialorrhea, etc.). The test is adaptive to the dynamics of the individual seizures: depending on the response (or lack of response) to an item, the next item to be tested is given, or an item has to be repeated. This way the output of the testing is optimized for the individual seizure. Due to the individual dynamics of each seizure, it is not expected to test all items for all seizures. The flow of the testing guides through the items applicable to the level of responsiveness of the individual patient, during the individual seizure.

The taskforce was established by the ILAE-CEA and the EEMA in 2014, who also approved the consensus statement. The battery was implemented in clinical practice in 10 European centers, and it was assessed on 250 seizures from 152 patients. In more than $90 \%$ of the included seizures, the battery was feasible.

The test was not found to be feasible in 17 cases. All these were very short seizures (myoclonus, short absence seizures and short focal seizures), where it was impossible to apply the testing procedure, in spite of the rapid reaction from the personnel, and there were no semiological features in the postictal period.

The standardized testing procedure shares many similarities with the local testing protocols that had been used in the participating centers as well as with the two previously published testing batteries ${ }^{7-9}$. The ictal bedside neuropsychological test procedure, published by Lux and colleagues, was not standardized throughout 
their study period and was less comprehensive compared to the European standardized procedure ${ }^{7}$. Nevertheless, the procedure was useful in identifying impairment of consciousness in patients with focal seizures $^{7}$. The Responsiveness in Epilepsy Scale (RES) and its modified version (RES-II) $)^{8-9}$ give scores for the degree of impairment of consciousness, and several studies demonstrated the usefulness in assessing consciousness during seizures ${ }^{11-13}$.

The procedure developed by the European taskforce is thus more comprehensive, aiming at demonstrating all possible semiological features. However, it is adaptive to the dynamics of the seizures, in order to increase feasibility and optimize efficiency. Ideally, each testing procedure should be tailored to the individual patient and seizure. However, this requires a high level of expertise and experience with testing patients during seizures that a number of EMU staff might lack. Indeed, experts from the taskforce in charge of developing the standardized battery felt that the main issue they experienced over the years when observing video-EEG recording of seizures from their or other centers was the lack of appropriate testing by the EMU staff. While epileptologists working in EMUs for many years have the capacity to run an optimally individualized testing without using any specific instrument, it happens that EMU staff usually in charge of that testing do not have similar level expertise and often lack proper training. In most European EMUs, patient's testing is performed by nurses, technicians or medical students ${ }^{6}$, with significant turnover of this staff in some EMUs leading them to rely on personnel with limited experience. Thus, the standardized battery primarily targets EMU staff that are expected to provide better assessment with such instrument than without, rather than highly experienced epileptologists. We believe that this might apply to all types of EMU recordings but again acknowledge that the current study only provide an evaluation of the feasibility of the testing battery and not its diagnostic performance. The standardized protocol also offers a structure upon which more individualized testing might be more easily applied. 
Implementing the standardized procedure for managing and testing patients during seizures in the EMU can potentially increase the quality of the data recorded during long-term video-EEG recordings and it can facilitate multicenter studies. Further studies are needed to assess the diagnostic added value of the testing battery.

\section{Key bullet points}

- The taskforce was appointed by the ILAE - Commission on European Affairs and the European Epilepsy Monitoring Unit Association.

- The taskforce developed a standardized battery for testing patients during and after seizures in Epilepsy Monitoring Units (EMUs).

- The feasibility of the battery was prospectively evaluated on 250 seizures, in 10 centers. It was feasible in $93 \%$ of the included seizures. 


\section{Acknowledgements}

We thank Bar-Adon T for help with the implementation of the procedure. We are grateful to the EMU staff who participated in the validation of the procedure: Skaarup C, Kjærgård H, Jensen TK, Eriksen JP, Kristiansen KV, Krogsgård Pico S, Eskelund Johansen AB, Bach Justesen A, Martinussen NI, Kliban A, Edelvik A, Charling C, Karlsson A, Petersson I-M, Milkevičienè V, Taffin F, Kengbo Y.

\section{Disclosure}

Dr. Trinka has acted as a paid consultant to Eisai, Ever Neuropharma, Biogen Idec, Medtronics, Bial, Shire and UCB and has received speakers' honoraria from Bial, Eisai, GL Lannacher, GlaxoSmithKline, Boehringer, Viropharma, Actavis, Teva, Newbridge and UCB Pharma. Dr. Trinka has also received research funding from UCB Pharma, Biogen-Idec, Red Bull, Merck, the European Union, FWF Österreichischer Fond zur Wissenschaftsförderung, and Bundesministerium für Wissenschaft und Forschung. Dr. Dobesberger has received speaker honoraria from Gerot-Lanach and travel support from Eisai, GlaxoSmithKline and Neurodata $\mathrm{GmbH} /$ Micromed Austria. Dr Rheims has has act as a paid consultant to EISAl, has received speakers' honoraria from EISAI, UCB Pharma and Advicenne and has received research funding from EISAI. Dr. Beniczky has received speaker honoraria from UCB and travel support from Elekta. The other co-authors declare no conflict of interest.

We further confirm that we have read the Journal's position on issues involved in ethical publication and affirm that this report is consistent with those guidelines. 
ILAE statement: This report was written by experts selected by the International League Against Epilepsy (ILAE) and was approved for publication by the ILAE. Opinions expressed by the authors, however, do not necessarily represent official policy or position of the ILAE.

Figure 1: Summary of the testing battery, with keywords

Supporting information 1: Test items recommended depending on the age of acquisition

Supporting information 2: Double-stimulus testing 


\section{REFERENCES}

1. Cascino GD. Clinical indications and diagnostic yield of video-electroencephalographic monitoring in patients with seizures and spells. Mayo Clin Proc 2002;77:1111-20.

2. Smolowitz JL, Hopkins SC, Perrine T, et al. Diagnostic utility of an epilepsy monitoring unit. Am J Med Qual 2007;22:117-22.

3. Velis $\mathrm{D}$, Plouin $\mathrm{P}$, Gotman J, et al. Recommendations regarding the requirements and applications for long-term recordings in epilepsy. Epilepsia 2007;48: 379-84.

4. Alving J, Beniczky S. Diagnostic usefulness and duration of the inpatient long-term video-EEG monitoring: findings in patients extensively investigated before the monitoring. Seizure 2009;18:470-3.

5. Blume WT, Lüders HO, Mizrahi E, et al. Glossary of descriptive terminology for ictal semiology: report of the ILAE task force on classification and terminology. Epilepsia. 2001;42:1212-8.

6. Rubboli G, Beniczky S, Claus S, et al. A European survey on current practices in epilepsy monitoring units and implications for patients' safety. Epilepsy Behav 2015; 44: 179-184.

7. Lux S, Kurthen M, Helmstaedter $\mathrm{C}$, et al. The localizing value of ictal consciousness and its constituent functions: a video-EEG study in patients with focal epilepsy. Brain. 2002;125:2691-8.

8. Yang L, Shklyar I, Lee HW, et al. Impaired consciousness in epilepsy investigated by a prospective responsiveness in epilepsy scale (RES). Epilepsia. 2012;53:437-47.

9. Bauerschmidt A, Koshkelashvili N, Ezeani CC, et al. Prospective assessment of ictal behavior using the revised Responsiveness in Epilepsy Scale (RES-II). Epilepsy Behav. 2013;26:25-8.

10. Berg AT, Berkovic SF, Brodie MJ, et al. Revised terminology and concepts for organization of seizures and epilepsies: report of the ILAE Commission on Classification and Terminology, 2005-2009. Epilepsia. 2010; 51:676-85. 
11. McPherson A, Rojas L, Bauerschmidt A, et al. Testing for minimal consciousness in complex partial and generalized tonic-clonic seizures. Epilepsia. 2012;53:e180-3.

12. Cunningham C, Chen WC, Shorten A, et al. Impaired consciousness in partial seizures is bimodally distributed. Neurology. 2014;82:1736-44.

13. Touloumes G, Morse E, Chen WC, et al. Human bedside evaluation versus automatic responsiveness testing in epilepsy (ARTiE). Epilepsia. 2016;57:e28-32. 